\title{
A new Hybrid Infection model optimization Algorithm
}

\author{
Zhi-jie Zhang \\ College of Computer Sci \& Tech, Southwest University for Nationalities, Chengdu, Sichuan, 610041, \\ China \\ e-mail: cdznf@126.com
}

Key words: Nature-Inspired, Global search, Local Search, infection mechanism.

Abstract: In this paper, a new nature-inspired optimization algorithm, based on the prevalent mechanism of infectious diseases, called Hybrid Infection model optimization Algorithm (HIA), was designed. The HIA algorithm mimic the transmission mechanism of infectious diseases, needs neither the gradient information nor the continuity of the search space. The comparison testing results of were showed that the new HIA optimization algorithm could works more efficient than some optimization algorithms in some cases.

\section{Introduction}

Optimization is one important research area of mathematical application. The most mainly optimize processing of optimization is using some methods to search the target solution space, under one constraint condition or more constraint conditions, for one special purpose or more special goals, to get the best or the worst solution or some specific max/min objects.

There are some typical optimization questions: Traveling Salesman Problem (TSP)、 Scheduling Problem (SP)、Knapsack Problem (KP)、Bin Packing Problem (BP)、 Max Clique Problem (MCP)etc., all of these optimization questions have very important theory research values and application research values. All of optimization questions with exactly objects could be defined according to non-linear constraint conditions[1]:

$$
\begin{aligned}
\underset{x \in \Re^{n}}{\max \text { imize } \min \text { imize }:} & f_{i}(x), \quad x=\left(x_{1}, x_{2}, \ldots, x_{n}\right)^{T} \in \mathfrak{R}^{n} \\
\text { Subject to: } & \phi_{j}(x)=0,(j=1,2, . ., M), \\
& \psi_{k}(x) \geq 0,(k=1,2, \ldots, N),
\end{aligned}
$$

The $f(x) 、 \phi_{i}(x)$ and $\psi_{j}(x)$ in $(1.1)(1.2)(1.3)$ are scalar functions of row vector. The every $x_{j}$ in $x=\left(x_{1}, x_{2}, \ldots, x_{n}\right)^{T}$ could be called the design variables, or the main variables, or the dominant variables, in this paper the $x_{j}$ were unify called as the main variable/variables s .

The optimization algorithm is one kind of search algorithm that using specific tactics to found the optimal solution or the best solution、 the second solution v the second best solution or the alternative solution, in one or more specially solution space, under some certainly constraint conditions or without any constraint conditions[2].

The optimization algorithm, could be classed into many different types: the global search algorithm and the local search algorithm[3][4][5]; the constraint search algorithm and the unconstraint search algorithm[6]; the multi-object search algorithm and single-object search algorithm[7]; the deterministic search algorithm 、 the stochastic search algorithm and the hybrid/mixture search algorithm[8][9],etc.. 


\section{Nature-inspired method}

With the emergence of the genetic algorithm, the modern nature-inspired algorithm, as a kind of new meta-heuristic search method, has come into being in 1972. The nature-inspired algorithm is easy to solve the complex optimization problem that hard to deal with by the method of traditional optimization tech, such as engineering optimization、 business planning, course arrangement、 TSP、MCP、 path planning, etc.

For its superior performance, the nature-inspired method is widely used in various kinds of optimization problems. At the same time, more and more new hybrid search algorithms based on nature-inspired emerge at the historic moment, such as: Simulated Annealing algorithm (SA) 、 Particle Swarm Optimization algorithm (PSO) 、Ant Colony Optimization algorithm (ACO) 、 Harmony Search algorithm (HS) 、 Cuckoo Search algorithm (CS), etc. All of these new algorithms provide more new and better methods to solve more diverse difficultly hard-optimization problems.

These new nature-inspired meta-heuristic search algorithms, could quickly obtained the approximately solutions meet the requirement of accuracy, could meet the requirement of computing time, could solve the NP-hard problem and get the approximate solution, could solve a variety of difficultly hard-optimization problem and get the approximate solution on time[10][11].

So the new nature-inspired search algorithm with or without meta-heuristic tech, could be used to solve the NP-hard problem、 hard-optimization problem for application of optimization[12][13]. The designs of hybrid nature-inspired search algorithm, are usually based on some kind of natural phenomenon of things by observation, and get the mathematical model of the natural phenomenon by mathematical abstraction.[14][15]

Then the math model of new algorithm should be converted into search and optimization algorithm step by step. In the end, the Hybrid Infection-based Nature-Inspired Algorithm was proposed and tested. The next, the design and implementation method of a new Hybrid search algorithm based on nature-inspired was introduced, the kind of Hybrid natural heuristic search algorithm based on infectious disease model, was named Hybrid Infection-based Nature-Inspired Algorithm (HIA).

\section{Infection search Algorithm}

At present, in the area of Mathematical Biology, the model of infectious diseases has been researched for many years[16][17][18]. On the one hand, the model for a variety of infectious diseases, combined with the historical statistical data, more statistical analysis had been done, On the other hand, for the establishment of mathematical models and prediction of infectious diseases, more and more mathematical methods were introduced.

The SIR model belong to the simple discrete time model of infectious diseases, many other epidemic models could be deduced from SIR, such as without heal or temporary recovery concept is introduced, the SIR should become into SIS; when the total number of target sample could be changed and individual classification concept of different ages was introduced, the SIR should become into the Leslie model[19][20][21]. So the SIR model was chose as the mathematical model of the new hybrid natured-inspired optimization algorithm[22][23].

In HIA, the brownian motion was used as one-dimensional motion formula, then expanded it to two-dimensional motion formula.

The HIA algorithm init parameters setting needed to meet conditions as following:

$$
S_{\text {num }}+I_{\text {num }}+R_{\text {num }}=N_{\text {num }} \text {; }
$$




$$
\begin{aligned}
& 0 \leq \omega_{s}=S_{n u m} / N_{n u m} \leq 1 ; \\
& 0 \leq \omega_{i}=I_{n u m} / N_{n u m} \leq 1 ; \\
& 0 \leq \omega_{r}=R_{\text {num }} / N_{\text {num }} \leq 1 ; \\
& \omega_{s}+\omega_{i}+\omega_{r}=1 .
\end{aligned}
$$

Therefore, the system init parameters as following: $\omega_{\mathrm{s}}=1 、 \omega_{\mathrm{i}}=0 、 \omega_{\mathrm{r}}=0$ The end parameters of system as following : $\omega_{\mathrm{s}}=0 、 \omega_{\mathrm{i}}=1 、 \omega_{\mathrm{r}}=0 \quad$ or $\quad \omega_{\mathrm{s}}=0 、 \omega_{\mathrm{i}}=0 、 \omega_{\mathrm{r}}=1 \quad$ or $\omega_{\mathrm{s}}=0 、 \omega_{\mathrm{i}}=\alpha 、 \omega_{\mathrm{r}}=1-\alpha$.

The other needed determined parameters: the total period of infection $\mathrm{T}$; the total sample of Group N; the total number of susceptible individuals $S_{n u m}$; the total number of infected individual $I_{\text {num }}$; The total number of individuals with immunity mechanic $R_{\text {num }}$; Contact coefficient $\beta$; the coefficient of was infected and after contact $\gamma$; Contact transmission coefficient $\mathfrak{R}_{0}$; ratio of Leave and join the total group of individual $b$. For initial value of each parameter, depending on the type of optimization problem be initialized. The total period of infection $\mathrm{T}$, stand for total cycles times operated by HIA Algorithm, is experience parameters depending on the type of problem, by the calculate method $T=$ Counter $=1 / \Delta_{i}$ and was set as 100 times.

The total sample of Group $\mathrm{N}$ was set as 1000 , decided by the scope of the solution space. The $S_{\text {num }} 、 I_{\text {num }}$ and $R_{\text {num }}$, at first, $S_{\text {num }}=N 、 \mathrm{I}_{\text {num }}=0 、 \mathrm{R}_{\text {rnum }}=0$, then the next generation $S_{\text {num }} 、 I_{\text {num }}$ and $R_{\text {num }}$ depended on $\beta 、 \gamma 、 \mathfrak{R}_{0}$ and $\mathrm{b}$, the init value of HIA algorithm $\gamma=1 、 b=0 、 \beta=\mathfrak{R}_{0}$ 、 $\mathfrak{R}_{0}=3 、 \alpha=0.3$.

\section{Hybrid Infection model optimization Algorithm, HIA}

\section{Begin}

Objective function $f(x), \quad x=\left(x_{1}, x_{2}, \ldots \ldots, x_{n}\right)^{T}$

Initialize the S、I、R population, get $\omega_{s}=i_{s} 、 \omega_{i}=i_{i} 、 \omega_{r}=r_{i}$;

Initialize best solution-set $\mathrm{N}$ and the size of $\mathrm{N}$;

Initialize parameters: $\beta, \gamma, \mathrm{b}, \alpha$;

Initialize iteration counter $\mathrm{c}$, and set iteration increment $\Delta i$;

While $\left(b \beta>(\gamma+b)\left(b-b \gamma-b^{2}\right)\right)$ or $(\mathrm{c}<\max$ number of iterations $\left.)\right)$

Generate new solutions by infectious mechanism

if $(f(x)$ is better than every-element in $\mathrm{N})$

update the best solution-set $\mathrm{N}$

else

Generate new solutions by Susceptible mechanism

\section{end if}

renew all $\omega_{s}=i_{s}-\Delta_{i} 、 \omega_{i}=i_{i}+\alpha \Delta_{i} 、 \omega_{r}=r_{i}+(1-\alpha) \Delta_{i} ;$

renew all $\beta, \gamma, \mathrm{b}, \alpha$;

end while

end

Fig 1 pause code of the HIA

The filtration mechanism of evaluation function was designed by this method: if the terminating conditions are not satisfied, the evaluation function should pick out the most suitable 
solution candidate set and as the seeds of next round of iteration. The evaluation function for $S_{t+1}$ 、 $I_{t+1}$ and $R_{t+1}$ as following:

$$
\text { num }_{\text {average }}=\sum_{i=1}^{m} I_{t}
$$

$\rho=f\left(s_{i}\right) /$ num $_{\text {average }}$;

If $\rho>1$, the solution of $s_{i}$ belong to $I_{t+1}$; if $\rho<1$, the solution of $s_{i}$ belong to $R_{t+1}$; if $\rho=1$, the solution of $s_{i}$ should belong to $I_{t+1}$ according to a random probability $\sigma$, else the solution of $s_{i}$ belong to $r_{t+1}$ according to a random probability $(1-\sigma)$.

The original dynamic constraints of HIA algorithm $0<\beta b\left(1-1 / \mathfrak{R}_{0}\right)<b \Re_{0}$ could be carried out by analyzing the following Jacobian matrix, if the limiting conditions of Jacobian matrix $b \beta>(\gamma+b)\left(b-b \gamma-b^{2}\right)$ could be meet, then, the HIA algorithm always could found appropriate solution. At the same time, another terminal condition decided by the variable of the total period of infection $\mathrm{T}$. The most simple method of terminal condition could designed by this formula:

$$
T=\text { Counter }=1 / \Delta_{i}
$$

Another terminal condition is that the counter of HIA had reached predetermined times of iterations, or, the optimal solution of HIA algorithm without any improvement after the set times of iterations had been reached.

\section{Test function and experiments}

There are many benchmark test functions in literature, and they are designed to test the Performance of optimization algorithms. Any new optimization algorithm should also be validated and tested against these benchmark functions. All of test results should be compared and analyzed as following table 1 , table 2 .

Test function 1: De Jong's fuction

$f(x)=\sum_{i=1}^{n} x_{i}^{2} ;-5.12 \leq x_{i} \leq 5.12, i=1,2, \ldots, n$; the mini value lied in $f\left(x_{i}\right)=0, x_{i}=0$, $i=1,2, \ldots, n$.

Test function 2: Axis parallel hyper-ellipsoid function

$f(x)=\sum_{i=1}^{n}\left(i \times x_{i}^{2}\right) ;-5.12 \leq x_{i} \leq 5.12, i=1,2, \ldots, n, x_{i}=0$, the mini value lied in $f\left(x_{i}\right)=0$, $i=1,2, \ldots, n$.

Test function 3: Easom's function

$f(x)=-\cos (x) \cos (y) \exp \left(-(x-\pi)^{2}-(y-\pi)^{2}\right) ;-100 \leq x_{i} \leq 100, i=1,2, \ldots, n ;$ when $x_{i}=0$, the mini value lied in $-1, \quad i=\pi, \pi, \ldots, \pi$.

Test function 4: Rosenbrock's function

$f(x)=\sum_{i=1}^{n-1}\left[100\left(x_{i+1}-x_{i}^{2}\right)^{2}+\left(1-x_{i}\right)^{2}\right] ; \quad-2.048 \leq x_{i} \leq 2.048, i=1,2, \ldots, n$, when $x_{i}=0$, the mini value $f\left(x_{i}\right)=0, \quad i=1,2, \ldots, n$.

Test function 5: Ratrigin function

$f(x)=10 n+\sum_{i=1}^{n-1}\left[x_{i}^{2}-10 \cos \left(2 \pi x_{i}\right)\right] ; \quad-5.12 \leq x_{i} \leq 5.12, \quad i=1,2, \ldots, n ; \quad x_{i}=0, \quad$ the mini value $f\left(x_{i}\right)=0, \quad i=1,2, \ldots, n$.

The test hardware environment of HIA algorithm is proposed as following: notepad IBM thinkpad x61, Intel(R) Core (TM) 2 Duo CPU, T7500, main frequency 2.2GH, memory2G; 
and, Matlab R2011b be used as Simulation software. The total statistical time of HIA algorithm that found the optimum solution or the approximate solution, was expressed as Time(seconds/s). The Accuracy Rate was used as the average time index that gets the same global optimal solution or found the best approximate solution deviation less than 3 percent after run 60times. The standard PSO algorithm and the Simulated Annealing Algorithm were used to compare with HIA algorithm. The results of compared were listed as following (Table 1 Table 2):

\begin{tabular}{|c|c|c|c|c|}
\hline \multirow{2}{*}{ Function } & \multicolumn{2}{|c|}{ PSO } & \multicolumn{2}{|c|}{ HIA } \\
\hline & Time(s) & Accuracy Rate & Time(s) & Accuracy Rate \\
\hline Function 1 & 42.6762 & $100 \%$ & 43.0288 & $100 \%$ \\
\hline Function 2 & 42.6877 & $100 \%$ & 42.8423 & $100 \%$ \\
\hline Function 3 & 41.2156 & $100 \%$ & 46.2066 & $100 \%$ \\
\hline Function 4 & 56.6264 & $97 \%$ & 52.0006 & $81 \%$ \\
\hline \multirow[t]{2}{*}{ Function 5} & 58.2192 & $85 \%$ & 58.4392 & $80 \%$ \\
\hline & \multicolumn{2}{|c|}{ Table 1: } & A compare & $\mathrm{PSO}$ \\
\hline \multirow[t]{2}{*}{ Function } & \multicolumn{2}{|c|}{$\mathbf{S A}$} & \multicolumn{2}{|c|}{ HIA } \\
\hline & Time(s) & Accuracy Rate & Time(s) & Accuracy Rate \\
\hline Function 1 & 43.1817 & $100 \%$ & 43.0288 & $100 \%$ \\
\hline Function 2 & 42.9373 & $100 \%$ & 42.8423 & $100 \%$ \\
\hline Function 3 & 43.5123 & $100 \%$ & 46.2066 & $100 \%$ \\
\hline Function 4 & 54.5567 & $100 \%$ & 52.0006 & $81 \%$ \\
\hline Function 5 & 54.9924 & $94 \%$ & 58.4392 & $80 \%$ \\
\hline
\end{tabular}

Table 2: The test results of HIA compared with SA

In table 1, the parameter Settings of PSO are as follows: the particle number $n=20$, the number of iterations is 100 beta $=0.5$, gamma $=0.7$. In table 2 , the parameter Settings of SA are as follows: the initialization temperature is 1 , the iteration stop temperature is $1 \mathrm{e}-10$, the cooling factor for alpha $=0.9$, the energy $E$ is $1 \mathrm{e}-5$, the maximum number of iterations is 500 .

The experimental results show that HIA algorithm can quickly generate more random candidate source distribution, and can quickly move towards their own local best or worst value. The HIA algorithm has good convergence and can quick search to the global optimal solution with fewer times of iterations. For some optimization instances, the HIA algorithm has faster speed for search better approximate solution or search the best solution more precisely.

\section{Conclusions}

For imitating the spread mechanism of infectious diseases, the HIA algorithm needs the spread transmission way of disease and the incremental iteration movement of the every generation new sources of infection.

At the same time, the concept of effective radius of influence, the infection factors etc. were introduced into the HIA algorithm, and all these concepts indeed increase search speed that found the candidate solution set around the target neighborhood.

The experimental results show that HIA algorithm can quickly generate more random candidate source distribution, and can quickly move towards their own local best or worst value. The HIA algorithm has good convergence and can quick search to the global optimal solution with fewer times of iterations. For some optimization instances, the HIA algorithm has faster speed for search 
better approximate solution or search the best solution more precisely.

\section{References}

[1].Polëìak B T. Introduction to optimization[M]. Optimization Software, Publications Division (New York), 1987.

[2].Papadimitriou C H, Yannakakis M. Optimization, approximation, and complexity classes [J]. Journal of computer and system sciences, 1991, 43(3): 425-440.

[3].Lourenço H R, Martin O C, Stützle T. Iterated local search[J]. International series in operations research and management science, 2003: 321-354.

[4].Lourenço H R, Martin O C, Stützle T. Iterated local search: Framework and applications[M] // Handbook of Metaheuristics. Springer US, 2010: 363-397.

[5].Lourenco H R, Martin O, Stützle T. A beginner's introduction to iterated local search[C] // Proceedings of MIC'2001-Meta-heuristics International Conference. 2001,1: 1-6.

[6].Jussien N, Lhomme O. Local search with constraint propagation and conflict-based heuristics[J]. Artificial Intelligence, 2002, 139(1): 21-45.

[7].Freuder E C, Quinn M J. Taking Advantage of Stable Sets of Variables in Constraint Satisfaction Problems [C] //IJCAI. 1985, 85: 1076-1078.

[8].Deb K. Multi-objective optimization[J].Multi-objective optimization using evolutionary algorithms, 2001: $13-46$.

[9].Laumanns M, Rudolph G, Schwefel H P. Mutation Control and Convergence in Evolutionary Multi-Object Optimization[J]. HT014601767, 2001.

[10].Mahdavi M, Fesanghary M, Damangir E. An improved harmony search algorithm for solving optimization problems[J]. Applied mathematics and computation, 2007, 188(2): 1567-1579.

[11].Wolpert D H, Macready W G. No free lunch theorems for search[R]. Technical Report SFI-TR-95-02-010, Santa Fe Institute, 1995.

[12].Wei W, Li C M, Zhang H. Deterministic and random selection of variables in local search for SAT[J]. Solver description,SAT competition, 2007, 2007.

[13].Hansen E A, Zilberstein S. LAO*: A heuristic search algorithm that finds solutions with loops[J]. Artificial Intelligence, 2001, 129(1): 35-62.

[14].Shi L, Olafsson S, Chen Q. A new hybrid optimization algorithm[J]. Computers \& Industrial Engineering, 1999, 36(2): 409-426.

[15].Fonseca C M, Fleming P J. An overview of evolutionary algorithms in multiobjective optimization[J]. Evolutionary computation, 1995, 3(1): 1-16.

[16].Murray J D. Mathematical biology[M]. springer, 2002.

[17].Murray J D. Mathematical biology[M]. Berlin: Springer, 1993.

[18].Rashevsky N. Advances and applications of mathematical biology[M]. Chicago, 1940.

[19].Weisstein E W. Kermack-McKendrick Model[J]. MathWorld--A Wolfram Web Resource. Retrieved, 2009, 16.

[20].Štěpán J, Hlubinka D. Kermack-McKendrick epidemic model revisited[J]. Kybernetika, 2007, 43(4): 395-414.

[21].Boccara N, Cheong K. Automata network SIR models for the spread of infectious diseases in populations of moving individuals[J]. Journal of Physics A: Mathematical and General, 1992, 25(9): 2447.

[22].Brauer F. Some simple epidemic models[J]. Mathematical Biosciences and Engineering, 2006, 3(1): 1.

[23].Anderson R M. Discussion: the Kermack-McKendrick epidemic threshold theorem[J]. Bulletin of mathematical biology, 1991, 53(1): 3-32. 